\author{
Lassâad Oueslati \\ Université de Tunis \\ Boulevard du 9 Avril 1938 \\ 1007 Tunis, Tunisia \\ lassaadoueslati2020@gmail.com \\ https://orcid.org/0000-0001-9226-5687
}

\title{
L'interprétation des unités phraséologiques entre combinatoire interne et emploi co-textuel : le cas des unités adverbiales polylexicales
}

\begin{abstract}
We will study, in this paper, the semantic functioning of phraseological adverbial units. How can they create meaning within their external combinations? What relationship do they have with the units that surround them? Do they impose semantic constraints on the units constituting their co-text? What are the factors of semantic variation of these units? By switching meaning, do these units change their function? We will attempt to answer these questions by describing a corpus of adverbial units according to the methodological approach of the theory of the three primary functions.

Keywords: cotext/context, phraseology, conceptual content, semantic content, unilexical, hetero-entity/self-entity
\end{abstract}




\section{Introduction}

Depuis une vingtaine d'années, les linguistes ont mis au point une série de critères permettant de décrire les unités polylexicales ${ }^{1}$ (UP). Dans cette description, ils ont focalisé sur la combinatoire interne. Ils ont eu recours à des critères génériques applicables à toutes les catégories syntaxiques ${ }^{2}$. Ces critères ont affiné les taxinomies de ces unités. Ils ont permis de distinguer les unilexicales des plurilexicales, les opaques des transparentes; etc.

Outre le contenu sémantique des SF, ces descriptions focalisent sur leur fonctionnement syntaxique : appartenance catégoriel et fonctions syntaxiques. En ce qui concerne l'étude sémantique, ces travaux cherchent à montrer que le sens de l'UP peut être la synthèse du sens des constituants ou, au contraire, un sens non-compositionnel. Bien que certains linguistes disent que l'UP fonctionne exactement comme une UM catégoriellement marquée et pourvue d'un sens, ils ont négligé l'impact du cotexte sur l'interprétation de ces unités.

Nous tenterons d'étudier l'influence de l'environnement textuel de l'UP adverbiale dans son interprétation. Nous rappellerons, en premier lieu, la double combinatoire des SF. Nous montrerons, en second lieu, l'interaction entre l'expression phraséologique et son cotexte dans le décodage. Nous appliquerons, en dernier lieu, cette démarche aux expressions phraséologiques adverbiales prises dans l'environnement textuel.

2. Les unités phraséologiques : la double combinatoire Avant de parler de la combinatoire de l'unité phraséologique, nous en fournissons, au préalable, une définition précise. Il s'agit d'une expression qui intrigue, vu son figement variable. Frath et Gledhill (2005) qualifient l'unité phraséologique de mystérieuse. «Son

\footnotetext{
${ }^{1}$ Nous citons à ce propos M. Gross (1986), G. Gross (1996), Mejri (1997; 2000; 2003; 2008; 2011) etc.

${ }^{2}$ Ces critères sont la polylexicalité, l'opacité sémantique, le blocage des propriétés transformationnelles, la non-actualisation des éléments constitutifs, le degré et la portée du figement, la non-insertion, le blocage des paradigmes synonymiques et l'étymologie.
} 
mystère, écrivent-ils, réside dans son figement variable, qui la distingue des mots simples et des phrases discursives. Elle intrigue en raison de l'habitude que nous avons de considérer les phrases et les syntagmes comme des constructions syntaxiques dans lesquelles des mots viennent s'insérer». Elle peut aller, ainsi, d'une unité lexicale (UL) à une unité phrastique et même à une unité textuelle passant par l'unité syntagmatique. Bally fut le premier à parler de ce concept ${ }^{3}$, et à en proposer une typologie. Il en distingue en effet deux types: les «unités phraséologiques indécomposables»dont le sens est noncompositionnel; et les unités lexicales dont les composants présentent des «affinités», les uns pour les autres. Ce phénomène concerne ainsi ses «séries phraséologiques». Les séries d'intensité, comme chaleur suffocante, grièvement blessé, etc., sont prototypiques.

Dépassant le point de vue de Bally, Legallois et Tutin (2013) ont essayé d'étendre le champ de la phraséologie pour examiner, outre les collocations, les séquences discursives telle que la parémiologie. Le croisement de données a affiné les critères définitoires des unités phraséologiques et a permis des classifications plus rigoureuses. Une expression phraséologique se définit principalement par le figement. Ce dernier est le dénominateur commun des locutions, des expressions figées, des expressions idiomatiques, des synthèmes, des phrasèmes, etc. Il est défini lui-même par une panoplie de propriétés élaborées principalement par G. Gross (1996) et S. Mejri (1998). Outre la polylexicalité, nous trouvons la non-compositionnalité, la fixité syntaxique, le blocage des propriétés transformationnelles, etc. L'application de ces critères définitoires a abouti à une discrimination entre, d'un côté, les unités indécomposables, entièrement figées, et de l'autre, les unités semi-figées. Étant donné que ces UP fonctionnent tout comme les UM, l'accent a été mis sur les deux combinatoires, interne et externe. D'ailleurs, à ce propos, Mejri écrit que «(...) la double combinatoire est une caractéristique essentielle dont on ne peut faire l'économie lors de la description des SF» (Mejri, 2008, p. 59). Aussi la description de la double combinatoire s'avère-t-elle

\footnotetext{
${ }^{3}$ Dans son ouvrage Traité de stylistique française (1921).
} 
nécessaire pour voir l'importance du cotexte dans l'interprétation des unités lexicales phraséologiques.

2.1. La combinatoire interne des unités phraséologiques La combinatoire interne d'une UL présuppose sa polylexicalité. En effet, le terme «combinatoire» renvoie à un agencement syntaxique entre des constituants de la même unité. Ces constituants ne sont que des morphèmes indépendants concaténés, jouant ainsi un rôle syntactico-sémantique, permettant de passer de la pluralité à l'unité globale. Tel n'est pas le cas pour les morphèmes dont la concaténation forme une UM. Soit les deux exemples suivants :

(1) Luc est incompréhensible.

(2) Luc est au chômage.

L'unité incompréhensible est composée de trois morphèmes dépendants : in-, la racine compréhens- et -ible. Étant dépendant, aucun de ces morphèmes ne peut fonctionner comme un mot autonome. La formation d'un mot dérivé nécessite la concaténation d'au moins deux morphèmes de façon à ce que l'UM obtenue soit insérable dans le discours. Dans ce cas précis, pour parler d'une UL, il faut partir de la concaténation des morphèmes deux et trois : la racine compréhens- et le suffixe -ible, la concaténation entre le premier morphème et la racine prédicative ne donnant pas lieu à un mot insérable dans le discours: *incompréhens. La formation de ce mot relève de la dérivation lexicale.

Quant au deuxième cas de figure, nous relevons la concaténation des morphèmes autonomes formant un syntagme: Prép. + Art. $+N=$ $\grave{a}+L e+C h o ̂ m a g e$. Composée de morphèmes indépendants, cette unité est le fruit de l'agencement des unités lexicales. Sa formation, contrairement à la précédente, relève plutôt de la syntaxe. Dans ce cas, nous parlons d'une combinatoire interne entre les constituants d'un syntagme. La différence entre les deux cas nous conduit à distinguer une UM d'une autre, UP, étant donné que, dans leur combinatoire externe, elles fonctionnent comme des prédicats adjectivaux. 
Dans ces deux cas de figure, la morphologie et la syntaxe se relaient. La combinatoire interne des UM relève de la morphologie dérivationnelle, alors que celle des UP relève plutôt de la combinatoire syntagmatique. À ce sujet, Mejri (2008, p. 264), apporte une explication de l'absence d'intérêt pour la morphologie des SF. Il l'impute à deux principales raisons: «(...) l'absence d'outil théorique» et «la rupture totale entre le morphologique et le syntaxique». Il propose une solution de nature à établir une complémentarité entre la morphologie et la syntaxe. Il écrit : «C'est en intégrant la morphologie polylexicale dans l'étude du lexique qu'on mettrait fin au cloisonnement entre les deux processus complémentaires de formation de mots. La langue peut avoir recours à l'un des deux processus pour former des unités à partir de la même base». Pour exemple, il donne la racine prédicative retrait- capable de donner à la fois une UM retraite/retraité et une UP, à la retraite.

Notre objectif est de réfléchir sur la combinatoire interne des unités phraséologiques dont la formation peut être décrite en termes syntaxiques. Les travaux sur la phraséologie ont abouti à deux principaux types d'UP : les unilexicales et les plurilexicales. Le critère permettant de discriminer ces deux types est de nature syntacticosémantique. Il touche à la fois au degré de figement de l'unité en question et à la globalisation de son sens.

En ce qui concerne l'unité unilexicale, elle est composée des constituants n'admettant aucune restructuration propre à la combinatoire libre. Tel est le cas en (3) et (4) (Mejri, 2008, p. 267) :

(3) Je souhaite en tout cas, qu'on aborde lors de congrès les sujets de fond ( $L e$ Monde 1993, cité par Mejri)

(4) Ces données ont été ensuite regroupées au niveau de chaque académie, afin d'établir un diagnostic d'ensemble et de déterminer une politique d'action. Et c'est la deuxième phase en cours» (Le Monde 1993, cité par Mejri)

Ces deux cas de figure de fond et en cours fonctionnent comme une UM indécomposable. Pour S. Mejri, la combinatoire interne de ces unités n'étant pas pertinente, leur description dans les dictionnaires ne doit focaliser que sur leur combinatoire externe. 
Quant à l'UP plurilexicale, elle se caractérise par un figement partiel. Il est possible de restructurer ses constituants en vue de mesurer leur degré de figement. C'est le cas de la locution mettre fin $\grave{a}^{4}$. Le figement de cette séquence étant relatif, elle peut admettre la transformation passive ou la construction impersonnelle :

(5) On a mis fin à ces atrocités

Fin a été mise à ces atrocités

Il a été mis fin à ces atrocités

À voir ces restructurations, nous pouvons penser que les transformations syntaxiques ne témoignent pas seulement d'une certaine liberté au niveau de la combinatoire, mais aussi de la naissance d'un nouveau sens ou la disparition du sens initial de la locution. En effet, la description de la combinatoire externe est nécessaire pour tenir compte du comportement syntaxique de l'UP plurlexicale et de son contenu sémantique qui peut, selon le cotexte, s'affaiblir ou se renforcer.

2.2. La combinatoire externe des unités phraséologiques

Un mot se définit par son appartenance catégorielle. Or pour rattacher un mot quelconque à une catégorie syntaxique, la morphologie, seule, demeure insuffisante dans la mesure où, à l'exception de la catégorie verbale, toujours reconnaissable, n'importe quelle autre UL peut verser dans une autre catégorie syntaxique, l'emploi phrastique de l'unité étant le seul déterminant. La description des UP se heurte à ce problème. En effet, ces unités peuvent avoir la structure de surface sous forme d'un syntagme prépositionnel (SP) tels qu'à la carte, de mèche, avec acuité, etc. Elles peuvent avoir également la structure d'un syntagme nominal (SN) : tête-bêche, marché conclu, modestie mise à part, les mains dans la poche, etc. Par conséquent, le sens et la combinatoire externe de l'UP sont déterminants dans la précision de sa catégorie grammaticale, dans sa distribution à l'intérieur de la phrase et dans la précision de son contenu sémantique. Combinée au cotexte, l'UP peut se voir changer de sens ou se pourvoir d'une

\footnotetext{
${ }^{4}$ Nous empruntons cet exemple à Mejri (2008, p. 267).
} 
nouvelle charge sémantique. Sur le plan syntaxique, on distingue depuis quelques années (Mejri, 1998) les auto-entités et les hétéroentités.

Ces deux types de SF montrent que le syntaxique et le sémantique sont liés dans la formation des UP. C'est un processus s'appuyant à la fois sur le contenu catégoriel et le contenu conceptuel des UL. En ce qui est du contenu catégoriel, l'unité ne change pas de partie de discours en se formant. La catégorie prototypique est celle des verbes (casser sa pipe). Étant, à l'origine, un syntagme verbal, cette locution forme une UP sans changement catégoriel. Devenue une UL figée, cette séquence garde tout de même un certain degré de variation flexionnelle. Sa fixité moins importante que celle du second type (voir infra) dans la mesure où le transfert se fait dans la même partie du discours, celle du verbe. L'élément tête, le verbe, permet, grâce aux flexions, d'ancrer l'unité dans le discours. Ainsi, ces unités autoentité, n'ayant pas de problème au niveau catégoriel, posent problème au niveau conceptuel.

Le second type, celui des unités hétéro-entités, regroupe des UP versées dans une autre partie du discours (adjectivaux, nominaux, adverbiaux, etc.). Que l'UP soit ainsi éloignée de sa catégorie syntaxique de départ est un signe de fixité forte (Mejri, 1998). En effet, la séquence subit, dans son emploi discursif une recatégorisation, sans aucune marque morphologique. Par exemple, l'UP à tête reposée est formée d'une préposition, à, qui verse le SN étendu tête reposée dans la catégorie adverbiale. Mais le problème n'est pas aussi simple. En effet, une SF telle qu'à la mode n'est que le résultat d'une re-catégorisation. Cependant, amputée de son environnement textuel, elle ne porte aucune marque morphologique de son éventuelle catégorie syntaxique. Seule sa distribution dans la phrase permet de le faire :

(6) Léa est à la mode.

(7) Léa s'habille à la mode.

S'agissant du contenu conceptuel des SF, il est intimement lié au comportement syntaxique des UP. 
3. L'interprétation des unités phraséologiques à la lumière des deux combinatoires : interne et externe

Composée de plusieurs morphèmes lexicaux indépendants, une UP pose problème quant à son contenu sémantique. Son sens est-il la synthèse du sens de tous ses composants ou il s'agit d'un sens nouveau différent du sens compositionnel ? Comment peut-on interpréter une UP ? Quel est l'impact du cotexte sur son sens ? Ces questions se justifient par le signifiant pluriel de l'UP. Pour G. Greciano et S. Mejri, il s'agit de globalisation ou de synthèse sémantique, pour Perrin (2011), du caractère bifacial de l'UP. Cette UP peut exprimer un contenu compositionnel ou non-compositionnel, les éléments déterminants dans son interprétation étant à la fois le contenu sémantique des constituants et son environnement textuel. Nous revenons sur ces deux facteurs sémantiques en étudiant le sens intrinsèque des UP en premier lieu et le sens résultant de leur combinatoire externe en second lieu.

3.1. Le contenu sémantique des unités phraséologiques

L'opacité sémantique, critère du figement, implique logiquement la transparence. $\mathrm{Vu}$ la polylexicalité de toute séquence phraséologique, le calcul du sens de cette séquence pose problème. En effet, il s'agit de voir si le sens d'une expression polylexicale est compositionnel ou non. Si la séquence phraséologique est constituée d'un signifiant pluriel, il est légitime de penser que cette unité est polysémique. Les points de vue à ce propos sont nombreux. Nous en retenons deux : celui de L. Perrin (2011) et celui de S. Mejri (1998; 2011; 2017). Le premier parle de l'ambivalence du contenu sémantique d'une SF, le second du dédoublement.

S'agissant de Perrin, il pense que l'unité phraséologique admet généralement deux lectures. Son postulat est que cette unité a nécessairement deux sens : un ascendant et un autre descendant. Le sens ascendant est « construit originel »; c'est le produit d'une lecture linéaire tenant compte de la concaténation des composants de la 
séquence. C'est le cas par exemple des expressions telles que casser sa pipe ou prendre une veste en (8) et (9) :

(8) Max a cassé sa pipe

(9) Max a pris une veste

Dépourvus d'éléments cotextuels capables de désambiguïser leur signification, ces deux exemples admettent une interprétation littérale, ce qui autorise, bien entendu, toutes les transformations syntaxiques possibles pour une séquence libre. Quant au sens descendant, il est défini comme «le sens lexical émergent». Le rapport entre les deux types de sens est régi par le degré de figement de l'expression phraséologique. C'est pour cela que «plus une expression est figée, selon Perrin, plus son sens codé descendant est dominant, et moins son sens ascendant est libre et accessible». Par conséquent, l'expression phraséologique s'inscrit dans un processus de lexicalisation. Dès que ce processus prend fin, le sens descendant domine et fait disparaitre le sens ascendant. Pour illustrer ce propos, Perrin cite l'expression métaphorique Prendre (ou saisir, attraper) la balle au bond. Le sens ascendant, issu d'une métaphore vive, signifie «avec dextérité à une situation critique», alors que le sens descendant, issu de l'expression figée, signifie «réagir par surprise et opportunément à une situation favorable». Même si le sens descendant gouverne cette expression, son sens ascendant, métaphorique, reste en veilleuse et par conséquent susceptible d'être réactivé par l'environnement textuel du moment que l'image métaphorique de prise de balle n'est pas morte.

Mejri, quant à lui, parle d'une structure englobante dans laquelle plusieurs unités simples viennent verser pour constituer une unité complexe. La concaténation de ces unités simples forme une unité ayant sa propre signification, laquelle est différente de celle des composants. Partant de ce raisonnement, il montre que dans le cas des SF, il n'est plus question d'enchainement syntagmatique, substitué plutôt par « une superposition paradigmatique » (Mejri, 1998, p. 17). Partant du constat que «la construction du sens se fait dans l'orientation opposée à celle de la concaténation des formants ou des 
constituants » (Mejri, 1998, p. 17), Mejri explique le fonctionnement de la SF. Pour parler d'unité dans ce cas, il faut distinguer deux niveaux structurant la SF. Le premier correspond «aux signifiants initiaux qui lui servent de constituants morphologiques». Le second niveau donne lieu à une UP présentée comme « un tout globalisant et global». C'est ce qui a conduit Mejri à préférer la notion de dédoublement à celle de polysémie. En effet, employés dans une SF, les constituants perdent momentanément toute autonomie syntaxique ou sémantique pour servir un fonctionnement global de l'UL émergente. Découle de ce qui précède que la pluralité des signifiants dans une SF n'est pas l'indice d'une polysémie. La description linguistique d'une telle unité devrait, sur le plan sémantique, s'intéresser non seulement à son sens intrinsèque mais aussi à la variation de ce sens dans un emploi cotextuel, tout comme l'UM. L'environnement textuel installe une relation interactive avec la SF.

3.2. L'interprétation de l'unité phraséologique dans son environnement textuel

La conceptualisation d'une SF est un processus consistant à pourvoir l'UP d'un sens nouveau. Vu le décrochage référentiel des constituants de la SF, la conceptualisation aboutit à un nouveau sémème. En fait, la condensation sémantique de cette $\mathrm{SF}$ assure une rupture entre ce qu'exprime globalement le signifiant pluriel et de ce que peut exprimer chacun des constituants du syntagme interprété linéairement. Par exemple, la locution verbale casser sa pipe (exemple 8) est une UL auto-entité puisqu'elle ne change pas de catégorie syntaxique. Cette locution se caractérise par une superposition paradigmatique. Par conséquent, elle admet naturellement une double lecture : une linéaire et une autre globale. Bien que l'interprétation compositionnelle de SF ne soit pas très fréquente, son environnement textuel oriente l'interprétation vers une signification non compositionnelle. Le rôle du cotexte dans cette interprétation non compositionnelle est déterminant. Soit les exemples suivants :

(10) Madame Verterelle a cassé sa pipe, annonce-t-il. Dans la rue. Elle se promenait quand tout d'un coup, (...) elle a eu une attaque, elle s'est étalée, 
on l'a ramassée sur le trottoir, elle était morte. (QUENEAU R. - Le dimanche de la vie (1951:132). Frantext ${ }^{5}$

(11) (Il) est mort à l'heure qu'il est. (...) Tu es assez malin pour comprendre que les civils ont parfois eux aussi un peu de jugeote. Il est mort d'une sorte de choléra très méchant qu'on appelle le choléra sec (...) Or, j'ai un remède. (...) Si je vois clair, il n'aura pas été seul à casser sa pipe. Alors, viens me voir, je te donnerai de quoi te sauver.» GIONO J. - Le Hussard sur le toit (1951 : 530). Frantext

Dans ces deux extraits textuels, le cotexte permet d'orienter l'interprétation de la SF. En effet, nous assistons, dans les deux exemples, à une isotopie de la mort conditionnant l'interprétation globale de cette expression phraséologique. Par exemple, l'extrait (10) commence par annoncer que «Madame Verterelle a cassé sa pipe ». La suite de la séquence n'en est qu'une explication. Nous avons ainsi une série de prédicats retraçant les étapes précédant la mort : d'abord se promener, ensuite une attaque, puis ramasser sur le trottoir et enfin morte. L'argument commun de ces prédicats est Madame Verterelle. Elle est tantôt agent tantôt patient. Outre ces prédicats, l'emploi de l'adverbe temporel quand suivi de l'adverbe tout d'un coup rattache la SF à la classe sémantique des <événements>. Or, la lecture linéaire de casser sa pipe la rattacherait à la classe des <actions>, excluant ainsi l'adverbe événementiel, tout d'un coup.

L'exemple (11) illustre cette idée. Cependant, l'emploi de la séquence casser sa pipe intervient à la fin de l'extrait, ce qui lui permet de synthétiser le cotexte précédent. En effet, la première phrase annonce déjà la mort du personnage (il est mort à l'heure qu'il est). Les phrases suivantes expliquent la raison de cette mort (choléra). Ainsi, la SF serait une reprise anaphorique du prédicat verbal mourir, antécédent de cette expression anaphorique. L'interpréter littéralement introduirait une perturbation au niveau de la cohérence conceptuelle du texte.

${ }^{5}$ Tous les exemples, de 10 à 26, sont empruntés à la base textuelle en ligne, Frantext (cf. Biliographie). 
4. Les unités phraséologiques adverbiales

Les locutions adverbiales ont de nombreuses structures telles que $S N+S P$ (le doigt dans le nez, etc. ; $N+$ Conj. $+N$ (bec et ongles), $S N+S N$ (bras dessus bras dessous), $N+$ Prép. $+N$ (pied à pied; ventre à terre, etc.) ; $A d v$. $+A d j$. (tout droit), etc. Mais la structure la plus productive est Prép. $+S N$ telles que à tête reposée, au pied levé, à vol d'oiseau (Mejri, 2007, p. 194). Outre la globalisation sémantique et la possibilité d'avoir un dédoublement de l'interprétation, cette structure pose un problème syntaxique, celui de son appartenance à l'une des parties du discours. C'est pour cela que nous aborderons la problématique du rôle du cotexte dans l'interprétation des expressions phraséologiques sous deux aspects : le contenu catégoriel et le contenu sémantique de ces UP.

4.1. Cotexte et contenu catégoriel des séquences à structure : Prép. + SN

N'étant pas marquées par le suffixe adverbial -ment, les rattachant à une catégorie grammaticale bien explicite, les UP présentent une certaine souplesse quant à leur fonctionnement syntaxique. En effet, elles ont la même structure qu'une expansion nominale, notamment les compléments de nom, introduits systématiquement par une préposition. Elles ont également la même structure qu'un SP qui peut, entre autres, fonctionner comme un complément circonstanciel. Que les SF introduites par une préposition puissent être un adjectif ou un adverbe montre à quel point le figement est capable de remédier au déficit à la fois morphologique et syntaxique. Si le suffixe cantonne l'UL dans une seule partie du discours et par conséquent réduit son fonctionnement syntaxique à celui de la partie en question, l'UP, introduite par une préposition, peut avoir l'avantage de ne pas être syntaxiquement codée au préalable. Seul l'emploi textuel permet de nous éclairer sur sa nature, étape importante précédant l'identification de sa fonction syntaxique. Mejri (2007, p. 194) écrit : «les formations syntagmatiques présentent le double avantage de rapprocher les parties du discours et de favoriser un fonctionnement multiple grâce à leur forme différenciée». 
Pour comprendre cette ambivalence fonctionnelle, examinons les deux séquences: sous le manteau et à l'amiable. La première a la structure Prép. + Dét. $+N$. Cette structure correspond à n'importe quelle séquence libre sous la forme d'un SP. La préposition sous a une valeur locative certaine, tout comme les prépositions dans et sur. Ce sème locatif inhérent impose des contraintes syntaxiques sur le verbe qui précède ce SP. En effet, le verbe régissant ce dernier introduit par la préposition sous devrait exprimer une action d'emplacement exigeant un complément circonstanciel de lieu tels que mettre, cacher, glisser, enfoncer, etc. Les exemples sont nombreux :

(12) «(Il) jette le manteau vert sur ses épaules et met le sien à la place dans le coffre, (...); il y ajoute son chapeau qu'il enfonce sous le manteau d'un coup de poing ; puis il referme le tiroir.» HUGO V., Ruy Blas, (1838 : 416). Frantext

Ce complément de lieu peut bien évidemment être repris anaphoriquement par un pronom relatif composé, sous lequel. Il peut aussi être thématisé sans le moindre changement sémantique. Au nom manteau on peut ajouter des modifieurs du type de fourrure, etc. Or, dès qu'on a affaire à la séquence figée, sous le manteau, toute cette liberté syntaxique et par conséquent sémantique devient contrainte. Par exemple, dans les extraits (13) et (14), aucune restructuration n'est possible :

(13) «Je publiai sous le manteau un roman chaud qui m'offrit la voiture neuve (...). NOURISSIER F. - À défaut de génie (2000 : 331). Frantext

(14) On vendait Frison-Roche, l'héroïsme des hauteurs. (...). Des romans, des romans, les gens voulaient s'évader, ils en redemandaient, qui circulaient sous le manteau, comme Autant en emporte le vent. Troyat, Benoit, Vialar, c'était maigre. Sauf le sublime Moby Dick, sa traduction par Giono, trois fois réimprimé chez Gallimard», GARAT A.-M. - Pense à demain (2010 : 314). Frantext

Employée dans deux cotextes consécutifs, la SF sous le manteau perd toute liberté combinatoire pour devenir une UL unilexicale n'admettant ainsi aucune insertion ou modification. De plus, le verbe précédant cette expression n'implique pas nécessairement l'idée d'emplacement. Même s'il exprime des actions nécessitant un cadre 
spatial, cet espace ne peut en aucun cas correspondre à un espace couvert par un manteau. Une lecture littérale de «romans circulaient sous le manteau » ou «je publiai sous le manteau un roman chaud» serait incongruente. C'est ce qui plaide en faveur d'une SF dont le noyau nominal a subi un décrochage ${ }^{6}$ référentiel et tous les constituants ont perdu leur autonomie. Cela ramène la pluralité que représentait le SP à l'unité que représente la SF. Cette unité assure ainsi un fonctionnement adverbial. Sa distribution dans le cotexte phrastique obéit aux contraintes syntaxiques afférentes à l'adverbe. Dès que le cotexte permet, par exemple, d'ajouter un modifieur du substantif manteau, toute la séquence ne peut être interprétée que comme une SL, comme nous l'observons dans l'exemple (15) :

(15) «Denise est entrée. (...) Elle portait un manteau de fourrure et s'est serrée contre moi. (...). Sous le manteau de fourrure elle avait un chemisier...» MODIANO P. - Rue des Boutiques Obscures (1978 : 212). Frantext

S'agissant de la deuxième séquence phraséologique à l'amiable, elle est introduite par le dictionnaire Le Petit Robert (2016) comme une locution adverbiale. Or, le même dictionnaire fournit deux exemples contradictoires quant à leur appartenance catégorielle: «Un arrangement à l'amiable. Divorcer à l'amiable ». Si le deuxième exemple est un adverbe, le premier est un adjectival dans la mesure où il est épithète. Dans leur cas, le substantif arrangement et le verbe divorcer servent de cotextes immédiats déterminant la catégorie syntaxique de la même séquence. Les cotextes de nature textuelle servent de façon plus efficace à désambiguïser la nature grammaticale de cette séquence unilexicale. Soit les deux exemples suivants :

(16) « Il a un métier d'avenir : de la merde, il fera de l'or ;(...) Sauf qu'il n'a pas de patente. (...) Ce qui lui crée quelques soucis de fiscalité, et lui barre des marchés. Il est très tenté par une négociation à l'amiable : contre une régularisation de son affaire, il est prêt à solder son frère aîné (...) ».GARAT A.-M. - Pense à demain (2010 : 550). Frantext

(17) « - Lewenthal est venu me voir (..). (...) nous avions réglé à l'amiable la petite contrariété d'Oranienburg...» GARAT A.-M. - Pense à demain (2010 : 451). Frantext

${ }^{6}$ Voir Mejri (1994, pp. 111-122). 
Cette expression phraséologique est composée de la préposition (à) et de l'adjectif substantivé (amiable). Telle qu'elle est structurée, elle ne peut être confondue avec un SP, comme sous le manteau. Étant donné qu' elle constitue une SF unilexicale, elle peut avoir un fonctionnement adjectival ou adverbial. Si en (16), elle porte sur un substantif prédicatif, elle porte, en (17), sur le verbe régler. Dans son emploi adjectival, cette expression n'est pas prédicative. Elle n'est par conséquent pas déplaçable (*à l'amiable négociation), ce qui en fait un adjectif classifieur non prédicatif puisqu'elle sert à préciser le type du procès sur lequel elle porte, autrement dit le type de négociation. Sur ce plan, elle fonctionne syntaxiquement tout comme un adjectif relationnel. Son emploi en (17) illustre cette idée de classifieur. Son fonctionnement adverbial précise la classe sémantique du procès sur lequel elle porte (régler). Il s'agit en effet d'une façon particulière servant à résoudre, régler des conflits, des litiges, etc.

4.2. Interaction entre le contenu conceptuel de la séquence adverbiale et son cotexte d'emploi

Les adverbes peuvent exprimer différentes valeurs sémantiques, grammaticales ou modales. Ces valeurs ne peuvent être suffisamment explicites que dans un emploi discursif. L'environnement textuel s'avère déterminant dans l'interprétation du contenu de l'adverbial, et vice versa. La description de notre corpus nous conduit à nous rendre compte du fait que la relation entre le cotexte et l'adverbial est de nature phraséologique. À ce propos, nous distinguons deux cas de figure : dans le premier, c'est le cotexte qui explicite le contenu de l'adverbe. Dans le second, c'est la séquence adverbiale qui influence l'interprétation de l'environnement textuel.

Dans le premier cas, certains adverbiaux peuvent avoir deux interprétations, une littérale et donc compositionnelle et une autre opaque, non compositionnelle. C'est le cas par exemple de nombreux adverbiaux dont à coeur ouvert. Le sens de cette SF peut changer en fonction des éléments cotextuels. Observons les exemples suivants :

(18) «Ils ont cotisé pendant quarante ans, ils voient les opérations à cœur ouvert à la télévision, on leur balance (...) un nouveau traitement du 
Parkinson ou de la maladie d'Alzheimer, alors ils veulent du médicament, de la chirurgie, des radios pour leur mal de pied, des échographies pour leurs crises de foie, des scanners en couleur pour leurs maux de tête. » WINCKLER M. - La maladie de Sachs (1998 : 116). Frantext

(19) « À ce propos, il faut que je vous parle de ma rencontre avec votre ami Lucien Aillaud. (...). Pour moi, je désirais vivement, (...) avoir une conversation à cour ouvert précisément avec un garçon comme lui.» WEIL S. - CEuvres (1929) LETTRES À JEAN POSTERNAK 1937: 645). Frantext

(20) «La curiosité, d'abord, un désir fraternel ensuite, qui croît bientôt en désir passionné de s'exprimer à cœur ouvert, de s'enfuir étroitement unis hors de ce cercle infernal, (...).» HAVET M. - Journal 1919-1924 (2005:147). Frantext

En (18) et (19), à coeur ouvert fonctionne comme un adjectival portant respectivement sur un substantif, opération et conversation. Leur contenu sémantique oriente leur interprétation. Il s'avère que nous avons deux adverbiaux homonymes. Pour preuve, l'adjectival (18) est employé comme un classifieur indiquant le type d'opération chirurgicale subie. Tout le cotexte relevant de la langue de spécialité des médecins oriente cette interprétation vers une lecture quasi linéaire. En effet, à cœur ouvert indique à la fois le type d'opération et l'organe opéré. Le champ sémantique relevant du cotexte (traitement, Parkinson, maladie, Alzheimer, médicament, la chirurgie, échographies, crises de foie, scanners, maux de tête, etc.) aide le lecteur à décoder le texte.

Cependant, le même adjectival, employé en (19) devient opaque. Portant sur un prédicat nominal conversation, il exprime ainsi la franchise et la sincérité. Tout le cotexte d'emploi conduit le lecteur vers une interprétation non compositionnelle. Qui plus est, seul cet emploi de l'adjectival admet un emploi adverbial. L'exemple (20) illustre cette idée. L'adverbial à cour ouvert s'emploie avec des prédicats verbaux appartenant à la classe des prédicats de <communication>. L'adverbial à pieds joints est un autre cas de figure, qui, en dehors d'un cotexte d'emploi, admet une lecture compositionnelle :

(21) «Le petit brun a poussé un cri aigu et il a sauté à pieds joints à travers la fenêtre ». MANCHETTE J.-P. - Que d'os ! (1976 : 85). Frantext 
Avec la précision locative (à travers la fenêtre) et verbe (sauter), l'adverbial renvoie à une posture physique lors du saut. Par conséquent, nous pouvons le paraphraser sans incongruence par un groupe gérondival, à l'instar du Petit Robert (2016) : «en gardant les pieds joints». Or tel n'est pas le cas de l'emploi du même adverbial dans un cotexte plutôt tropique. Dès que l'un des arguments change de classe sémantique, on passe du propre au figuré comme en (22) :

(22) «(...) Je déteste la littérature en demi-teintes. Je me concentre, je fais le vide, épaules rentrées, genoux serrés, je saute à pieds joints dans le texte. De nouveau, il me ravit. Je l'ai déjà lu dix fois. A la onzième, il me transporte. Sur le tapis volant, dans la machine à remonter le temps, bond formidable en arrière. » DOUBROVSKY S. - Le Livre brisé (1989 : 105). Frantext

Dans cet exemple, en dépit de la présence d'un argument sujet humain (je) et du verbe prédicatif (sauter), nous sommes amené à une interprétation non compositionnelle de la séquence adverbiale opaque. Ce changement de sens est imposé par un argument locatif abstrait (dans le texte). La paraphrase par pieds rapprochés n'est plus possible pour la même séquence adverbiale. Le cotexte large contribue ainsi dans cette interprétation non compositionnelle mettant l'accent sur un comportement face à un texte littéraire. Les prédicats suivants confirment cette posture intellectuelle (me ravit, me transporte/ sur le tapis volant, dans la machine à remonter le temps). Compte tenu de tous ces éléments lexicaux formant le cotexte, l'interprétation ne peut se faire qu'à partir du sens global, «sans hésiter», selon Le Petit Robert. Les exemples (23) et (24) illustrent bien cette idée :

(23) Et me voilà, à six ans, tombant à pieds joints dans un imbroglio d'adultère et de libertinage (...). CAPRI A. RUSSIE, $(1975: 38)$. Frantext

(24) « Je m'amusai méchamment à marcher à pieds joints sur son amour-propre» GOBINEAU A. de - Les Pléiades (1874 :176). Frantext

L'interprétation globale et non pas linéaire est favorisée principalement par l'argument N1. En (23), même si le prédicat verbal indique un déplacement et nécessite par conséquent un argument objet à valeur locative, l'UP adverbiale à pieds joints ne peut être paraphrasée littéralement. La raison du blocage d'une telle 
interprétation est le sens de cet argument N1. En (23) et (24), les deux $\mathrm{SN}$, consécutivement un imbroglio d'adultère et de libertinage et son amour propre sont introduits par deux prépositions à valeur locative, dans et sur. L'incompatibilité sémantique entre la valeur locative de ces prépositions et le sens abstrait des syntagmes nominaux crée une relation conflictuelle, laquelle est génératrice de métaphore ${ }^{7}$.

Dans le deuxième cas de figure, il est question des adverbiaux qui influencent l'interprétation du cotexte. Les cas de figure sont très nombreux. Par exemple, certains adverbiaux peuvent conférer au prédicat une valeur aspectuelle qu'il n'exprimait pas seul. À ce propos, nous pouvons citer l'adverbial tout le temps :

Cette histoire, tu la racontes tout le temps.

Si l'on supprime l'adverbe, le présent du verbe raconter change de valeur. Sans adverbe, le présent a une valeur énonciative et peut être ambigu dans la mesure où il peut avoir une valeur injonctive. Cependant, avec la valeur aspectuelle durative de l'adverbial, le verbe raconter, dont le mode d'action est le perfectif, exprime l'aspect itératif. De nombreux adverbiaux confèrent au prédicat sur lequel ils portent une valeur aspectuelle qu'il n'exprime pas dans d'autres cotextes (cf. Borillo, 1991). Cette situation concerne de nombreux adverbiaux tels que d'arrache-pied, de plus en plus, à temps, en un clin d' eil, etc.

Parallèlement, certains adverbiaux, outre la modification de la valeur aspectuelle inhérente du prédicat, parviennent même à convertir le prédicat dans une autre classe sémantique à laquelle il n'appartenait pas dans la langue. C'est le cas de ce que nous avons appelé «adverbe convertisseurs de classes sémantique» (Oueslati, (2018, pp. 81-92). Certains font partie de cette classe. Nous en examinons un seul cas, en un clin d'eeil.

Cet adverbial se caractérise par son opacité sémantique. Il renvoie à un intervalle très court. C'est pour cela qu'il est compatible avec des prédicats dont le mode d'action exprime le ponctuel tel que

\footnotetext{
${ }^{7}$ Pour approfondir la question, cf. M. Prandi (2016).
} 
disparaitre. Cette quasi-absence de durée interne du procès fait qu'il soit incompatible avec tout ce qui exprime la durée comme les états. Or, à voir cet adverbial s'employer avec, non seulement, un prédicat d'état, mais aussi avec le présent de l'indicatif ou l'imparfait, nous relevons le pouvoir qu'il a à convertir un prédicat d'état dans la classe des événements. Soit les exemples suivants :

(25) «Les bagages (...) étaient (...) reniflés par deux chiens tenus en laisse et muselés, dont l'absence de réaction au passage de la mallette (...) permettait d'espérer qu'au moins elle ne contenait pas de stupéfiants. Puis, quand Max se fut installé dans le petit avion, celui-ci se mit en mouvement à toute allure, gagnant en un clin d'œil son altitude et sa vitesse de croisière, (...) » ECHENOZ J. - Au piano (2003 : 178). Frantext

(26) «Mais la nuit était finie. J'étais restée debout toute la nuit. J'étais si contente d'avoir tenu jusqu'à la fin de la nuit que je n'éprouvais aucune fatigue. (...) Deux kapos arrivaient, sifflaient, et en un clin d'œil tout le monde était debout. » DELBO Ch. - Auschwitz et après II (1970 : 171). Frantext

En (25), la première phrase comporte, outre l'imparfait à valeur descriptive, une série de prédicats d'état, «reniflés», "permettait d'espérer » «contenait». Nous sommes face à une description de la scène générale où se déroule le procès. La phrase suivante introduit le passé simple qui marque le passage de la description à la narration. Parallèlement au passé simple, l'adverbial en un clin d'oil favorise une lecture événementielle du prédicat altitude, introduit par le support gagner. Ce prédicat appartenant à la classe sémantique d'<état> se prête à une interprétation événementielle dans la mesure où l'adverbial permet de voir dans le prédicat altitude un changement d'état à valeur aspectuelle télique. La même situation peut être illustrée en (26). En effet, ce dernier est également descriptif, d'où l'emploi de l'imparfait et des prédicats d'état. Dans ce passage, le même prédicat «debout», dans un premier emploi, appartient à la sous-classe de <posture> de l'hyperclasse <Etat>; et dans un deuxième emploi, se convertit à la classe des <événements> en exprimant <un changement d'état>. Cette conversion est assurée par la 
conjonction de coordination et à valeur consécutive et l'emploi de l'adverbial renforçant la conséquence, et donc le changement d'état ${ }^{8}$.

\section{Conclusion}

Nous avons essayé dans ce travail de montrer à quel point le cotexte est important dans l'interprétation des unités phraséologiques de façon générale et les unités adverbiales de façon particulière. Cependant, la notion de cotexte est réduite dans ce travail à un environnement textuel. Il concerne aussi bien les unités lexicales que les unités grammaticales, les temps verbaux et les adverbes de modalité. Cependant, certains facteurs extra-textuels peuvent orienter l'interprétation d'une séquence adverbiale et par conséquent tout le cotexte. L'énonciation peut avoir une grande influence sur l'interprétation d'un énoncé ou d'un discours. Elle peut permettre à un discours d'exprimer l'opposé de ce que disent ses mots. Une étude approfondie des éléments énonciatifs gagnerait à être menée pour mettre en lumière la dimension pragmatique dans la construction du sens.

\section{Bibliographie}

Bally, Ch. (1921). Traité de stylistique française. Heidelberg: Carl Winters Universitatsbuchhandlung

Borillo, A. (1991). La nature compositionnelle de l'aspect. Travaux de linguistique et de philologie, 29, 97-102.

Frath, P., \& Gledhill, Ch. (2005). Qu'est-ce qu'une unité phraséologique ? In C. Bolly, J-R. Klein, \& B. Lamirov (Eds.), La Phraséologie dans tous ses états. Actes du colloque "Phraséologie 2005", 13-15 oct. 2005. Cahiers de l'Institut de Linguistique de Louvain, 31/2-4, 11-25.

Gross, G. (1996). Les expressions figées en français et autres locutions. Paris: Ophrys.

Guimier, C. (1996). Les adverbes en français : le cas des adverbes en-ment. Paris: Ophrys.

Legallois, D., \& Tutin, A. (2013). Présentation : Vers une extension du domaine de la phraséologie. Langages, 189, 3-25.

${ }^{8}$ Remarquons qu'en dépit de l'emploi de l'imparfait et du prédicat d'état « debout », l'adverbial prend le dessus pour exprimer l'événement. 
Mejri, S. (1994). Séquences figées et expression de l'intensité. Essai de description sémantique. Cahiers de lexicologie, 65, 111-122.

Mejri, S. (1997). Le figement lexical, description linguistique et structuration sémantique. Tunis: Publications de la Faculté des Lettres de la Manouba.

Mejri, S. (1998). Structuration sémantique et variation des séquences figées. In S. Mejri, G. Gross, A. Clas, \& T. Baccouche (Eds.), Le figement lexical. Actes de la $I^{r e}$ Rencontre Linguistique Méditerranéenne RLM (pp. 103-112). Tunis: CERES.

Mejri, S. (1999). Unité lexicale et poylexicalité. Linx, 40, 79-93

Mejri, S. (2003). Introduction : polysémie et polylexicalité. Syntaxe \& sémantique, 5, 13-30.

Mejri, S. (2006). Polylexicalité, monolexicalité et double articulation. Cahiers de Lexicologie, 2, 209-221.

Mejri, S. (2007). Les adjectivaux ambivalents : morphologie et prédication. In S. Mejri (Ed.), À la croisée des mots. Hommages à Taïeb Baccouche (pp. 193-205). Sousse: Université de Sousse \& Université de Paris 13.

Mejri, S. (2011). Figement, collocation et combinatoire libre. In J.-C Anscombre, \& S. Mejri (Eds.), Le figement linguistique : la parole entravée (pp. 41-62). Paris: Honoré Champion.

Mejri, S. (2017). L'expression du contenu grammatical, entre morphosyntaxe et lexique. In C. Badiou-Montferrand, S. Bajric, \& Ph. Monneret (Eds.), Penser la langue. Sens, texte, histoire (pp. 293-307). Paris: Honoré Champion.

Mejri, S. (2008). La double combinatoire des séquences figées. In G. Gross, \& K. U. Schulz (Eds.), Linguistics, computer, science and language processing. Festschrift for Franz Guentner on the occasion of his $60^{\text {th }}$ birthday (Tributes) (pp. 259-270). London: College Publications

Oueslati, L. (2006). Les constructions adverbiales en français contemporain. Thèse à la carte. Villeneuve d'Ascq: ANRT.

Oueslati, L. (2015). Les adverbiaux classifieurs de prédicats de déplacement. Synergie Tunisie, 4, 35-50.

Oueslati, L. (2018). Adverbiaux appropriés et propriétés aspectuelles. In O. Soutet, S. Mejri, \& I. Sfar (Eds.), La phraséologie : Théories et applications (pp. 81-92). Paris: Honoré Champion.

Perrin, L. (2011), Figement, énonciation et lexicalisation citative. In J.-C. Anscombre, \& S. Mejri (Eds.), Le figement linguistique: La parole entravée (pp. 81-94). Paris: Honoré Champion.

Prandi, M. (2016). Les métaphores conflictuelles dans la création de concepts et de termes. Langue française, 189, 35-48. DOI: 10.3917/lf.189.0035.

Sources

Frantext, Retrieved from https://www.frantext.fr/repository/frantext. 